\title{
INVARIANT IMBEDDING AND RANDOM WALK
}

\author{
RICHARD BELLMAN
}

1. Introduction. Let us consider the lattice points lying between the integers $a$ and $b$ and a particle constrained to occupy only these sites and the positions $a$ and $b$ :

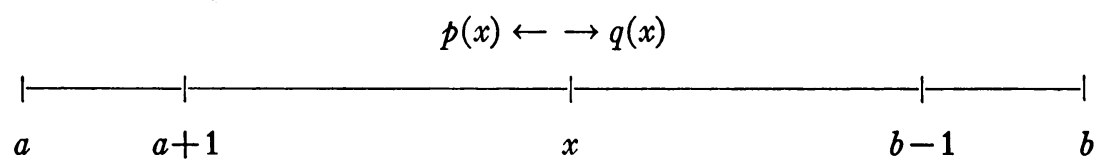

The classical random walk problem is that of studying the motion of a particle which when at $x$ has a probability of moving one unit to the left with probability $p(x)$ and one unit to the right with probability $q(x)=1-p(x)$.

Designating by $u(x)$ the probability that a particle starting at $x$ occupies $a$ before it occupies $b$, it is easily seen that

$$
u(x)=p(x) u(x-1)+q(x) u(x+1), \quad a<x<b,
$$

with the boundary conditions

$$
u(a)=1, \quad u(b)=0 .
$$

Although this system of linear equations determines the sequence $\{u(x)\}$, it is not the most convenient way to obtain these values. In a previous paper [1], it was shown that the functional equation technique of invariant imbedding could be used to obtain an interesting and meaningful representation of the solution of (1). In this paper, we wish to apply the same method to the study of a more general random walk process.

To simplify the algebraic details we shall consider where the particle can go one or two steps in either direction. It will be clear that similar methods can be applied to the case where any finite number of steps can be taken in either direction.

Consider the case where there is a continuous distribution of step sizes, $k(x, y) d y$. Then the equation for $u(x)$ corresponding to (1) is

$$
u(x)=\int_{-\infty}^{a} k(x, y) d y+\int_{\infty}^{b} k(x, y) u(y) d y, \quad a \leqq x \leqq b .
$$

One way of solving this linear integral equation of Fredholm type is to replace it by an approximating system of linear algebraic equa-

Received by the editors February 10, 1961. 
tions. An entirely different approach is to approximate to the continuous distribution by a discrete distribution and then to use the results that follow.

2. Invariant imbedding approach. Let us now consider the case where with assigned probabilities the particle can go one or two steps in either direction. The analysis that follows is based upon the simple idea that in order for a particle to escape to the left, i.e. land at $a$ or $a-1$ before occupying $b+1$ or $b$, it must necessarily land at $a+1$ or $a+2$ first.

We introduce the two functions

$u(a, x)=$ the probability of starting at $x$ and escaping to the left by landing at $a$,

$v(a, x)=$ the probability of starting at $x$ and escaping to the left by landing at $a-1$,

for $a<x<b$. We have

$$
\begin{gathered}
u(a, b)=v(a, b)=0, \\
u(a, a)=1, \quad v(a, a)=0 .
\end{gathered}
$$

From the remark of the first paragraph, it follows that

(3) $\quad v(a-1, x)=u(a+2, x) v(a-1, a+2)$

$$
+v(a+1, x) v(a-1, a+1) \text {. }
$$

Thus, for $x \geqq a+2$,

$$
\left(\begin{array}{c}
u(a, x) \\
v(a-1, x)
\end{array}\right)
$$

$$
=\left(\begin{array}{cc}
u(a, a+2) & u(a, a+1) \\
v(a-1, a+2) & v(a-1, a+1)
\end{array}\right)\left(\begin{array}{c}
u(a+2, x) \\
v(a+1, x)
\end{array}\right) .
$$

Introducing the matrix

$$
U(a, x)=\left(\begin{array}{cc}
u(a, x+1) & u(a, x) \\
v(a-1, x+1) & v(a, x)
\end{array}\right)
$$

for $x \geqq a$, we see that (4) yields the relation

$$
U(a, x)=U(a, a+1) U(a+2, x),
$$

for $x \geqq a+2$. 
We see then that the determination of $U(a, x)$ is reduced to the determination of $U(a, a+1)$ and $U(a, a)$ as functions of $a$.

3. $U(a, a+1)$. Let us introduce the elementary probabilities $p_{i}(x)=$ the probability of moving $i$ steps to the left when at $x$,

$$
i=1,2 \text {, }
$$

$q_{i}(x)=$ the probability of moving $i$ steps to the right when at $x$,

Then it follows in the usual fashion that

$$
i=1,2 .
$$

$$
\begin{aligned}
u(a, a+2)= & p_{2}(a+2)+q_{1}(a+2) u(a, a+3) \\
& +q_{2}(a+2) u(a, a+4), \\
u(a, a+1)= & p_{1}(a+1)+q_{1}(a+1) u(a, a+2) \\
& +q_{2}(a+1) u(a, a+3), \\
v(a-1, a+2)= & q_{1}(a+2) v(a-1, a+3) \\
& +q_{2}(a+2) v(1, a+4), \\
v(a-1, a+1)= & p_{2}(a+1)+q_{1}(a+1) v(a-a-1, a+2) \\
+ & q_{2}(a+1) v(a-1, a+3) .
\end{aligned}
$$

In the second and fourth of these relations we use the values of $u(a, a+2)$ and $v(a-1, a+2)$ obtained from the first and third. The result is

(3)

$$
\begin{aligned}
u(a, a+1)= & p_{1}(a+1)+q_{1}(a+1)\left[p_{2}(a+2)\right. \\
& \left.+q_{1}(a+2) u(a, a+3)+q_{2}(a+2) u(a, a+4)\right] \\
& +q_{2}(a+1) u(a, a+3), \\
v(a-1, a+1)= & p_{2}(a+1)+q_{1}(a+1)\left[q_{1}(a+2) v(a-1, a+3)\right. \\
& \left.+q_{2}(a+2) v(a-1, a+4)\right] \\
& +q_{2}(a+1) v(a-1, a+3) .
\end{aligned}
$$

Hence we may write

$$
\begin{gathered}
U(a, a+1)=\left(\begin{array}{cc}
p_{2}(a+2) & p_{1}(a+1)+q_{1}(a+1) p_{2}(a+2) \\
0 & p_{2}(a+1)
\end{array}\right) \\
+U(a, a+3)\left(\begin{array}{ll}
q_{2}(a+2) & q_{1}(a+1) q_{1}(a+2) \\
q_{1}(a+2) & q_{1}(a+1) q_{1}(a+2)+q_{2}(a+1)
\end{array}\right), \\
U(a, a+1)=P(a)+U(a, a+3) Q(a) .
\end{gathered}
$$

Using the relation 
(6)

$$
U(a, a+3)=U(a, a+1) U(a+2, a+3),
$$

the case $x=a+3$ of (6), we have, from (5),

$$
U(a, a+1)=P(a)+U(a, a+1) U(a+2, a+3) Q(a) .
$$

Hence

$$
U(a, a+1)=P(a)(I-U(a+2, a+3) Q(a))^{-1} .
$$

Since

$$
U(b-1, b)=\left(\begin{array}{ll}
u(b-1, b+1) & u(b-1, b) \\
u(b-1, b+1) & u(b-1, b)
\end{array}\right)=0,
$$

by means of (8) we can calculate $U(a, a+1)$ for all $a \leqq b-1$.

A similar calculation yields the corresponding recurrence relation for $U(a, a)$.

4. Discussion. In the same way we can discuss other quantities of interest, such as the expected duration of the process, time-dependent and angle-dependent processes for multidimensional regions, and so on; see $[2 ; 3]$.

The interesting point is that this technique affords a decomposition of the general random walk process completely analogous to that obtained in [2] for the simple one-step process.

\section{REFERENCES}

1. R. Bellman and R. Kalaba, Random walk, scattering and invariant imbeddingI: one-dimensional case, Proc. Nat. Acad. Sci. U. S. A. 43 (1957), 930-933.

2. - Invariant imbedding, random walk and scattering-II: discrete versions, J. Math. Mech. 9 (1960), 411-420.

3. R. Bellman, R. Kalaba and G. M. Wing, Invariant imbedding and mathematical physics-I: particle processes, J. Math. Phys. 1 (1960), 280-308.

Rand Corporation 